\title{
Is preoperative staging enough to guide lymph node dissection in clinically early gastric cancer?
}

\author{
Jia $\mathrm{Xu}^{1,2} \cdot$ Hui Cao ${ }^{2} \cdot J^{\prime}$-Young Yang ${ }^{1} \cdot$ Yun-Suhk Suh $^{1} \cdot$ Seong-Ho Kong ${ }^{1}$. \\ Se-Hyung Kim ${ }^{5}$ Sang-Gyun Kim ${ }^{6}$ Hyuk-Joon Lee ${ }^{1,3,4}$ - Woo-Ho Kim ${ }^{4,7}$. \\ Han-Kwang Yang ${ }^{1,3,4}$
}

Received: 27 November 2014/Accepted: 6 June 2015/Published online: 1 August 2015

(c) The International Gastric Cancer Association and The Japanese Gastric Cancer Association 2015

\begin{abstract}
Background Limited by the accuracy of preoperative staging, some cases of gastric cancer invading the muscularis propria (pT2) are underestimated as early gastric cancer (EGC) in the preoperative assessment. The aim of this present study was to determine prognostic factors and to propose indications for limited lymph node dissection in patients with clinically EGC (cEGC).

Methods Patients of cEGC $(n=2072)$ who were postoperatively diagnosed as $\mathrm{pT} 1(\mathrm{cT} 1 \mathrm{pT} 1, n=1858)$ and $\mathrm{pT} 2$
\end{abstract}

J. Xu and $\mathrm{H}$. Cao contributed equally to this work.

Electronic supplementary material The online version of this article (doi:10.1007/s10120-015-0512-1) contains supplementary material, which is available to authorized users.

Han-Kwang Yang

hkyang@snu.ac.kr

1 Department of Surgery, Seoul National University Hospital, Seoul, Korea

2 Department of General Surgery, Renji Hospital, Shanghai Jiaotong University School of Medicine, Shanghai, China

3 Department of Surgery, Seoul National University College of Medicine, 101 Daehang-Ro, Jongno-Gu, Seoul 110-744, Korea

4 Cancer Research Institute, Seoul National University College of Medicine, Seoul, Korea

5 Department of Radiology, Seoul National University College of Medicine, Seoul, Korea

6 Department of Internal Medicine, Seoul National University College of Medicine, Seoul, Korea

7 Department of Pathology, Seoul National University College of Medicine, Seoul, Korea
(cT1pT2, $n=214$ ) from 2005 to 2009 at Seoul National University Hospital were retrospectively analyzed.

Results There was no difference in 5-year survival rate between the cT1pT1 and cT1pT2 group $(95.5 \%$ vs. $92.5 \%, P=0.059)$, and both groups had better overall survival than $\mathrm{pT} 2$ patients who were preoperatively diagnosed as locally advanced gastric cancer (cT2-4pT2), whose 5-year survival rate was $78.0 \%(P<0.001)$. Multivariate analysis indicated lymph node metastasis (LNM) was the independent prognostic factor for cEGC $(P<0.001)$. In cEGC patients, three preoperative factors, including $\mathrm{N}$ stage by multidetector-row computed tomography (MDCT) $(P<0.001)$, preoperative histological type $(P<0.001)$, and tumor size $(P<0.001)$, were associated with LNM by multivariate analysis. Regarding the possibility of LNM, low-risk (4.4\%) and high-risk (17.3\%) groups were developed based on weighted scores of the aforementioned independent three variables. Among 52 patients in the low-risk group, the extension of LNM was limited to the perigastric area.

Conclusions Comprehensive evaluation based on MDCT, preoperative histological type, and tumor size is an effective method to predict LNM and guide tailored LN dissection for cEGC.

Keywords Early gastric cancer - Prognosis - Lymph node metastases - Lymphadenectomy

\section{Introduction}

Early gastric cancer (EGC) is defined as gastric cancer with lesions that have only invaded to the mucosa or submucosa, regardless of lymph node metastases (LNM). 
With the promotion and popularization of regular screening by endoscopy, as well as the emergence of high-resolution endoscopy, the diagnostic rate of EGC has been increasing year by year. Especially in Korea and Japan, with their high incidences of gastric cancer, the proportion of EGC among all treated gastric cancer cases has exceeded $60 \%$ [1]. Although radical gastrectomy and D2 lymph node dissection remain the standard surgical treatment for gastric cancer, certain limited treatment options for EGC, such as endoscopic submucosal dissection (ESD), function-preserving gastrectomy, and laparoscopic gastrectomy, have been accepted by increasing numbers of people because of their characteristics of faster postoperative recovery, better postoperative quality of life, and comparable therapeutic effect [2]. In addition, the current selections in lymph node dissection for EGC range from $\mathrm{D} 1$, to $\mathrm{D} 1^{+}$, to standard D2 dissections based on the preoperative assessment of $\mathrm{N}^{-}$staging [3]. However, compared to traditional surgery, the limited surgery has more stringent indications and may not be applicable to all patients with gastric cancer. Therefore, an accurate preoperative assessment of staging is particularly important. Currently, the clinical preoperative staging of gastric cancer primarily relies on the examinations of multidetector-row computed tomography (MDCT), esophagogastroduodenoscopy (EGD), and endoscopic ultrasonography (EUS). However, because of the small lesion size of EGC, some pathological changes cannot be recognized by the naked eye or displayed with CT or EUS. Cases of advanced gastric cancer (AGC) invading the muscularis propria (pT2) are commonly underestimated as EGC in the preoperative assessment of clinical practices [4]. It is not certain that limited lymph node dissection would change the prognosis of the pT2 patients who have been underestimated and treated as EGC. In addition, the current preoperative prediction of LNM still relies primarily on the change in the size of the lymph nodes in CT or EUS. The positive rate of LNM in EGC patients with LNM often does not increase along with size; thus, even intraoperative probing has difficulty in clearly revealing the metastases and infiltration of the lymph nodes [5]. Therefore, for clinical early gastric cancer (cEGC), it is not feasible to select limited lymph node dissection $\left(\mathrm{D} 1, \mathrm{D}^{+}\right)$or expanded lymph node dissection (D2) based solely on the size of the lymph nodes [6]. To solve this problem, we performed a retrospective analysis of the prognosis and the risk factors associated with LNM in cEGC patients to determine the prognostic factors and to propose indications for extended lymph node dissection for patients with cEGC in pathological $\mathrm{T} 1$ and $\mathrm{T} 2$ gastric cancer.

\section{Patients and methods}

\section{Study subjects and grouping}

The clinicopathological data and the follow-up records for a total of 3885 patients diagnosed with gastric cancer who underwent surgery at Seoul National University Hospital from January 2005 to December 2009 (the last follow-up was carried out in September 2013) were retrospectively analyzed. The hospital ethics committee had approved the use of the data (IRB No. H-1501-043-639). The cases that met the following inclusion criteria were selected. (1) The patients underwent preoperative EGD and MDCT examinations, with optional EUS examination, and the preoperative $\mathrm{T}$ staging was cT1, namely, cEGC. The diagnostic criteria of the preoperative $\mathrm{T}$ staging (clinical $\mathrm{T}$ stage, $\mathrm{cT}$ stage) by MDCT, EGD, and EUS was based on our previously published literature [7]. When the results of cT staging by different examinations were inconsistent, the relatively earlier $\mathrm{T}$ staging was selected as the final $\mathrm{cT}$ stage. (2) The patients were pathologically diagnosed with $\mathrm{T} 1$ or T2 gastric cancer according to the American Journal of Critical Care (AJCC) cancer staging handbook, 7th edition [8]. (3) The patients underwent $\mathrm{R} 0$ resection and at least D1 lymph node dissection with curative intention. The exclusion criteria were as follows: (1) patients who showed recurrent gastric cancer or distant metastases; (2) patients with synchronous gastric cancer or combined with other cancer; (3) patients who received neoadjuvant chemotherapy before the surgery. After the screening, 2072 cases that were pathologically diagnosed as pT1 and pT2 of cEGC were included in the research. These cases were divided into group A (cT1pT1, $n=1858)$ and group B (cT1pT2, $n=214$ ) according to the preoperative and postoperative T staging. Two hundred and sixty-seven cT2-4pT2 patients (with preoperative $\mathrm{T}$ staging of advanced gastric cancer and postoperative pathological diagnosis of $\mathrm{T} 2$ ) undergoing R0 surgery in the same period of time were denoted as group C. The comparison of group B (cT1pT2) to group A (cT1pT1) and to group C (cT2-4pT2) was performed in terms of clinical pathological findings and differences in the overall postoperative survival.

\section{Clinical and pathological data}

Clinical and pathological data of the included cEGC patients were collected. By univariate and multivariate analysis, the influencing factors of long-term survival in the patients with cT1pT1 (group A) and cT1pT2 (group $\mathrm{B})$, as well as the preoperative diagnostic indicators 
related to the LNM of cEGC, were investigated. The collected clinical and pathological data included patient demographics (age and gender), tumor characteristics (tumor size, location, gross and microscopic classification, and invasion of lymphatic vessels, blood vessels, and peripheral nerves), and the information related to LNM (preoperative CT and EUS diagnosis of LNM; postoperative pathological examination for the total number of lymph nodes, the number of metastatic lymph nodes, and the location of metastatic sites). In this study, the World Health Organization (WHO) histological tumor types were classified into well-differentiated (WD), moderately differentiated (MD), and poorly differentiated (PD). Papillary adenocarcinoma was classified as WD, and signetring cell adenocarcinoma and mucinous adenocarcinoma were classified as PD. The WHO types based on the preoperative endoscopic biopsy and postoperative pathological specimens were collected and compared. Based on the receiver operating characteristic (ROC) curve, the tumors were divided into the $<2.5 \mathrm{~cm}$ group and the $\geq 2.5 \mathrm{~cm}$ group according to the Youden index. The gross type of EGC was recorded according to the Japanese classification of gastric carcinoma [9] and divided into the elevated type (I, IIa, IIb) and the depressed type (IIc, III). In case of combined type, such as IIc + IIa, the tumor was classified as a depressed type (IIc). The tumors were divided into two subgroups for analysis according to the position of the tumor on the longitudinal axis (upper $1 / 3$, middle $1 / 3$, and lower $1 / 3$ ) and the circular axis (anterior, posterior, lesser curvature, and greater curvature) of the stomach. Detailed diagnostic criteria of LNM by preoperative CT and EUS were previously published [7]. The postoperative pathological staging of LNM was conducted using two staging methods, including the number of positive lymph nodes and the LNM site. The former method followed the TNM staging system of the AJCC cancer staging handbook, 7th edition [8], and the $\mathrm{N}$ staging was based on the number of pathologically positive lymph nodes detected after the surgery. Metastatic lymph nodes in which station numbers were identified was classified according to the Japanese classification of gastric carcinoma (JCGC) [9]. According to the LNM classification, patients with LNM were divided into two groups: metastases only in first-tier lymph nodes and metastases in lymph nodes of second-tier and higher, with or without first-tier metastases.

\section{Statistical methods}

The SPSS (version 19.0) statistical software package was used for data analysis. Overall survival (OS) was calculated using the Kaplan-Meier method, and the survival curve was plotted. The survival data were compared by the log-rank test. Cox proportional hazard models with variable selection procedures were used to investigate the multivariable association between covariates and survival. Univariate analysis was performed using the chi-square test and Fisher's exact probability test for analysis of risk factors for LNM. Multivariate analysis was performed using the logistic regression model. A risk model was constructed to stratify cEGC patients into different LNM risk groups based on factors that remained significant on multivariable analysis. A weighting method, which had been employed in previous studies by other investigators $[10,11]$, was applied to each variable in our analysis. The $\beta$-regression coefficients were applied to develop prognostic scores for each variable in the model, with higher scores indicating a greater probability of LNM. The score was calculated by dividing the regression coefficients by the smallest $\beta$-coefficient of the variables, then multiplying by 2.0 and rounding to the nearest whole number. $P$ values less than 0.05 indicated statistically significant differences.

\section{Results}

\section{Comparison of group B (cT1pT2) to group A (cT1pT1) and to group C (cT2-4pT2) in terms of clinical pathological findings and overall survival curve}

The comparison in clinical pathological findings of group B (cT1pT2, $n=214)$ to group A (cT1pT1, $n=1858)$ and to group C (cT2-4pT2, $n=267$ ) is shown in Table 1 . The LNM percentages of the patients in group B and group C were $31.8 \%$ and $49.4 \%$, respectively, with significant difference $(P<0.001)$. Although group $\mathrm{B}$ also had significantly higher LNM than group A, in group B the LNM occurred more often in N1 stage. Of the 66 patients with LNM in group B, 52 cases $(78.8 \%)$ were limited in N1 stage. In addition, the number of dissected lymph node in group B was significantly lower than group C $(P=0.005)$, but presented no differences compared to group A $(P=0.209)$. The overall survival curves of the three groups of patients with different preoperative and postoperative $\mathrm{T}$ stages were drawn and compared (Fig. 1). The postoperative 5-year survival rate of the patients in group B and group A were similar at $92.5 \%$ and $95.5 \%$, respectively, with no statistically significant difference $(P=0.059)$; In addition, the postoperative 5-year survival rates of the patients in group $\mathrm{B}$ were significantly higher 
Table 1 Comparisons of clinicopathological features among group A (cT1pT1), group B (cT1pT2), and group C (cT2-4pT2)

\begin{tabular}{|c|c|c|c|c|c|}
\hline Variables & Group A, $n=1858(\%)$ & Group B, $n=214(\%)$ & Group C, $n=267(\%)$ & $P$ value $^{\mathrm{a}}$ & $P$ value \\
\hline \multicolumn{4}{|l|}{ Age (years) } & 0.001 & $<0.001$ \\
\hline$<60$ & $951(51.2)$ & $135(63.1)$ & $108(40.4)$ & & \\
\hline$\geq 60$ & $907(48.8)$ & 79 (36.9) & $159(59.6)$ & & \\
\hline \multicolumn{4}{|l|}{ Gender } & 0.286 & 0.729 \\
\hline Male & $1235(66.5)$ & $150(70.1)$ & $191(71.5)$ & & \\
\hline Female & $623(33.5)$ & $64(29.9)$ & $76(28.5)$ & & \\
\hline \multicolumn{4}{|c|}{$\mathrm{N}$ stage by 7 th AJCC } & $<0.001$ & $<0.001$ \\
\hline No & $1717(92.4)$ & $148(69.2)$ & $135(50.6)$ & & \\
\hline N1 & $76(4.1)$ & $52(24.3)$ & $53(19.9)$ & & \\
\hline $\mathrm{N} 2$ & $48(2.6)$ & $11(5.1)$ & $54(20.2)$ & & \\
\hline $\mathrm{N} 3$ & $17(0.9)$ & $3(1.4)$ & $25(9.4)$ & & \\
\hline \multicolumn{4}{|c|}{ EGC gross type } & $<0.001$ & - \\
\hline I, IIa, IIb & 449 (26.3) & $28(14.3)$ & - & & \\
\hline IIc, III & $1258(73.7)$ & $168(85.7)$ & - & & \\
\hline \multicolumn{4}{|c|}{ Postoperative WHO type } & $<0.001$ & 0.640 \\
\hline WD & $489(26.3)$ & $20(9.3)$ & $32(12.0)$ & & \\
\hline MD & $628(33.8)$ & $80(37.4)$ & $99(37.1)$ & & \\
\hline $\mathrm{PD}$ & $740(39.9)$ & $114(53.3)$ & $136(50.9)$ & & \\
\hline \multicolumn{4}{|l|}{ Lauren type } & $<0.001$ & 0.317 \\
\hline Intestinal & $1123(61.4)$ & $98(46.2)$ & $138(53.1)$ & & \\
\hline Diffuse & $574(31.4)$ & $88(41.5)$ & $92(35.4)$ & & \\
\hline Mixed & $133(7.2)$ & $26(12.3)$ & $30(11.5)$ & & \\
\hline \multicolumn{4}{|l|}{ Tumor size } & $<0.001$ & $<0.001$ \\
\hline$<2.5 \mathrm{~cm}$ & $996(53.6)$ & $79(37.1)$ & $43(16.1)$ & & \\
\hline$\geq 2.5 \mathrm{~cm}$ & $861(46.4)$ & $134(62.9)$ & $224(83.9)$ & & \\
\hline \multicolumn{4}{|c|}{ Lymphatic invasion } & $<0.001$ & $<0.001$ \\
\hline Negative & $1623(87.5)$ & $141(66.2)$ & $87(48.3)$ & & \\
\hline Positive & $231(12.5)$ & $72(33.8)$ & $93(51.7)$ & & \\
\hline \multicolumn{4}{|c|}{ Venous invasion } & 0.003 & 0.012 \\
\hline Negative & $1821(98.3)$ & $202(94.8)$ & $158(87.8)$ & & \\
\hline Positive & $32(1.7)$ & $11(5.2)$ & $22(12.2)$ & & \\
\hline \multicolumn{4}{|c|}{ Perineural invasion } & $<0.001$ & 0.249 \\
\hline Negative & $1818(98.1)$ & $160(75.1)$ & $144(80.0)$ & & \\
\hline Positive & $35(1.9)$ & $53(24.9)$ & $36(20.0)$ & & \\
\hline \multicolumn{4}{|c|}{ Total number of examined LN } & 0.209 & 0.005 \\
\hline$\leq 30$ & $978(52.7)$ & $103(48.1)$ & $95(35.6)$ & & \\
\hline$>30$ & $879(47.3)$ & $111(51.9)$ & $172(64.4)$ & & \\
\hline \multicolumn{4}{|c|}{ Tumor location (longitudinal) } & $<0.001$ & 0.101 \\
\hline Upper $1 / 3$ & $159(8.8)$ & $33(16.5)$ & $43(16.8)$ & & \\
\hline Middle 1/3 & $496(27.6)$ & $85(42.5)$ & $85(33.2)$ & & \\
\hline Lower $1 / 3$ & $1143(63.6)$ & $82(41.0)$ & $128(50.0)$ & & \\
\hline \multicolumn{4}{|c|}{ Tumor location (circular) } & 0.207 & 0.287 \\
\hline $\mathrm{LC}$ & $734(40.1)$ & $88(41.5)$ & $93(36.9)$ & & \\
\hline GC & $236(12.9)$ & $19(9.0)$ & $37(14.7)$ & & \\
\hline AW & $412(22.5)$ & $43(20.3)$ & $50(19.8)$ & & \\
\hline PW & $449(24.5)$ & $62(29.2)$ & $72(28.6)$ & & \\
\hline
\end{tabular}

$E G C$ early gastric cancer, $W D$ well-differentiated, $M D$ moderately differentiated, $P D$ poorly differentiated, $L N$ lymph nodes, $L C$ lesser curvature, $G C$ greater curvature, $A W$ anterior wall, $P W$ posterior wall

${ }^{\text {a }}$ Compared between group B and group A

${ }^{\mathrm{b}}$ Compared between group B and group C 


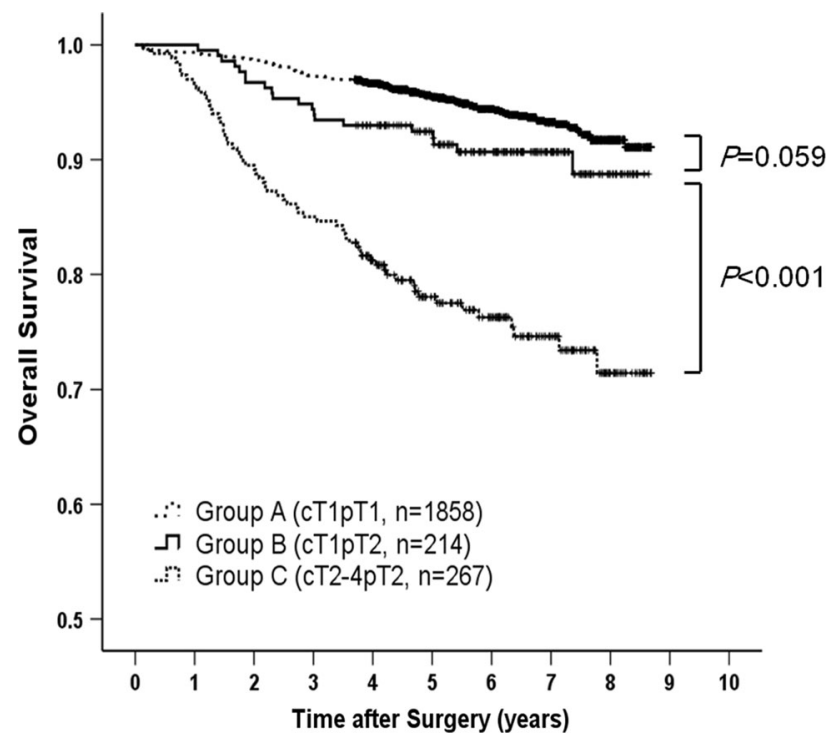

Fig. 1 Comparison of overall survival (OS) curves between group A (cT1pT1), group B (cT1pT2), and group C (cT2-4pT2)

than the $78.0 \%$ postoperative 5-year survival rate in group $\mathrm{C}(P<0.001)$

\section{Prognostic analysis in the cEGC patients}

The foregoing survival analysis confirmed that the cT1pT2 patients can be treated the same way as EGC in receiving limited lymph node resection. Therefore, we combined both cT1pT2 and cT1pT1 patients into one group to undergo the subsequent analysis. The univariate analysis showed that age, gender, LNM, lymphatic invasion, vascular invasion, and total number of examined LN were the relevant factors affecting the 5-year overall survival of cEGC (Table 2). The subsequent multivariate analysis of these prognostic factors showed that age, gender, and LNM were the independent risk factors for the prognosis of cEGC (Table 2).

\section{Analysis of the factors affecting LNM and preoperative prediction}

Based on the foregoing study, LNM is the important factor affecting the prognosis of cEGC patients. However, the present study showed that the diagnostic sensitivities of LNM in cEGC by preoperative MDCT and EUS were only $19.3 \%(40 / 207)$ and $2.9 \%(2 / 69)$, respectively (Table 3$)$. To improve the sensitivity of the preoperative prediction of LNM, the following univariate analysis using the preoperative diagnostic factors showed that the preoperative $\mathrm{N}$ staging by MDCT, preoperative WHO histological type, Lauren classification, tumor size, and tumor location
Table 2 Univariate and multivariate analysis of prognostic characters for patients with cEGC

\begin{tabular}{|c|c|c|c|}
\hline \multicolumn{4}{|c|}{ Univariate analysis } \\
\hline Variables & Number & 5 -year-survival rate $(\%)$ & $P$ value \\
\hline \multicolumn{3}{|l|}{ Age (years) } & $<0.001$ \\
\hline$<60$ & 1086 & 98.0 & \\
\hline$\geq 60$ & 986 & 92.0 & \\
\hline \multicolumn{3}{|l|}{ Gender } & $<0.001$ \\
\hline Male & 1385 & 94.0 & \\
\hline Female & 687 & 97.4 & \\
\hline \multicolumn{3}{|c|}{ T stage by 7 th AJCC } & 0.059 \\
\hline pT1 & 1858 & 95.5 & \\
\hline \multirow[t]{2}{*}{ pT2 } & 214 & 92.5 & \\
\hline & & & $<0.001$ \\
\hline \multicolumn{4}{|l|}{ LNM } \\
\hline $\mathrm{N}^{-}$ & 1865 & 96.1 & \\
\hline $\mathrm{N}^{+}$ & 207 & 86.6 & \\
\hline \multicolumn{3}{|c|}{ EGC gross type } & 0.097 \\
\hline I, IIa, IIb & 477 & 96.4 & \\
\hline IIc, III & 1426 & 94.9 & \\
\hline \multicolumn{3}{|c|}{ Postoperative WHO type } & 0.120 \\
\hline WD & 509 & 94.6 & \\
\hline MD & 708 & 94.0 & \\
\hline PD & 854 & 96.5 & \\
\hline \multicolumn{3}{|l|}{ Lauren type } & 0.101 \\
\hline Intestinal & 1221 & 94.3 & \\
\hline Diffuse & 662 & 96.5 & \\
\hline Mixed & 159 & 96.7 & \\
\hline \multicolumn{3}{|l|}{ Tumor size } & 0.215 \\
\hline$<2.5 \mathrm{~cm}$ & 1075 & 95.9 & \\
\hline$\geq 2.5 \mathrm{~cm}$ & 995 & 94.4 & \\
\hline \multicolumn{3}{|c|}{ Lymphatic invasion } & 0.003 \\
\hline Negative & 1764 & 96.1 & \\
\hline Positive & 303 & 90.5 & \\
\hline \multicolumn{3}{|c|}{ Venous invasion } & $<0.001$ \\
\hline Negative & 2023 & 95.5 & \\
\hline Positive & 43 & 81.1 & \\
\hline \multicolumn{3}{|c|}{ Perineural invasion } & 0.140 \\
\hline Negative & 1978 & 95.2 & \\
\hline Positive & 88 & 96.6 & \\
\hline \multicolumn{3}{|c|}{ Total number of examined LN } & 0.015 \\
\hline$\leq 30$ & 1081 & 94.1 & \\
\hline$>30$ & 990 & 96.3 & \\
\hline \multicolumn{3}{|c|}{ Tumor location (longitudinal) } & 0.363 \\
\hline Upper $1 / 3$ & 192 & 92.7 & \\
\hline Middle $1 / 3$ & 581 & 96.4 & \\
\hline Lower $1 / 3$ & 1225 & 95.2 & \\
\hline \multicolumn{3}{|c|}{ Tumor location (circular) } & 0.534 \\
\hline $\mathrm{LC}$ & 822 & 94.7 & \\
\hline GC & 255 & 95.5 & \\
\hline AW & 455 & 94.5 & \\
\hline
\end{tabular}


Table 2 continued

\begin{tabular}{|c|c|c|c|c|}
\hline \multicolumn{5}{|c|}{ Univariate analysis } \\
\hline \multirow{2}{*}{$\frac{\text { Variables }}{\text { PW }}$} & Number & \multicolumn{2}{|c|}{ 5-year-survival rate $(\%)$} & \multirow[t]{2}{*}{$P$ value } \\
\hline & 511 & 96.0 & & \\
\hline \multicolumn{5}{|c|}{ Multivariate analysis } \\
\hline & \multicolumn{2}{|c|}{ Risk ratio } & $95 \% \mathrm{CI}$ & $P$ value \\
\hline Age ( $\geq 60$ years) & \multicolumn{2}{|c|}{3.820} & $2.538-5.750$ & $<0.001$ \\
\hline Gender (male) & \multicolumn{2}{|c|}{2.040} & $1.308-3.180$ & 0.002 \\
\hline $\mathrm{LNM}\left(\mathrm{N}^{+}\right)$ & \multicolumn{2}{|c|}{2.711} & $1.785-4.118$ & $<0.001$ \\
\hline
\end{tabular}

$L N M$ lymph node metastases, $E G C$ early gastric cancer, $W D$ welldifferentiated, $M D$ moderately differentiated, $P D$ poorly differentiated, $L N$ lymph nodes, $L C$ lesser curvature, $G C$ greater curvature, $A W$ anterior wall, $P W$ posterior wall

(circular axis) were the predictive factors for LNM in cEGC (Table 3). Because the univariate analysis indicated that the probability of LNM for preoperative MD and PD was $11.4 \%$ and $12.2 \%$, respectively, and no significant difference between the two groups $(P=0.626)$, we combined the preoperative MD and PD patients in the subsequent multivariate analysis. The multivariate analysis of the relevant factors showed that the preoperative $\mathrm{N}$ staging by MDCT, preoperative WHO histological type, and tumor size were the independent risk factors for LNM (Table 4).

According to the different weights of the $\mathrm{N}$ stage by $\mathrm{CT}$, preoperative WHO histological type, and tumor size in the risk logistic regression model of LNM, these three variables were given different scores in the range of $0-4$ (Table 4). The sum of the scores of each variable reflects the risk of LNM for that patient. As the score becomes larger, the chance of the occurrence of LNM becomes greater (Table 4). The ROC curve was drawn for the diagnosis of LNM in cEGC based on the scoring criteria and compared with the ROC curves for the preoperative diagnoses of LNM by MDCT and EUS (Fig. 2). The area under the ROC curve of the diagnosis of LNM by the composite score was 0.728 , which was significantly higher than for the diagnoses by MDCT or EUS alone (0.591 and 0.511 , respectively). According to the Youden index of ROC curve, the cEGC patients were divided into the lowrisk group ( $<4$ points) and the high-risk group ( $\geq 4$ points) for LNM based on their composite scores, with a $74.7 \%$
Table 3 Univariate analysis of risk factors for lymph node metastases in patients with $\mathrm{cEGC}$

\begin{tabular}{|c|c|c|c|c|}
\hline Variables & Number & $\mathrm{N}^{-}(\%)$ & $\mathrm{N}^{+}(\%)$ & $P$ value \\
\hline Total, $n(\%)$ & 2072 & $1865(90.0)$ & $207(10.0)$ & \\
\hline \multicolumn{4}{|l|}{ Age (years) } & 0.509 \\
\hline$<60$ & 1086 & $973(89.6)$ & $113(10.4)$ & \\
\hline$\geq 60$ & 986 & $893(90.5)$ & $94(9.5)$ & \\
\hline \multicolumn{4}{|l|}{ Gender } & 0.107 \\
\hline Male & 1385 & $1257(90.8)$ & $128(9.2)$ & \\
\hline Female & 687 & $608(88.5)$ & $79(11.5)$ & \\
\hline \multicolumn{4}{|c|}{$\mathrm{N}$ stage by MDCT } & $<0.001$ \\
\hline $\mathrm{N}^{-}$ & 1932 & 1765 (91.4) & $167(8.6)$ & \\
\hline $\mathrm{N}^{+}$ & 140 & $100(71.4)$ & $40(28.6)$ & \\
\hline \multicolumn{4}{|c|}{$\mathrm{N}$ stage by EUS } & 0.106 \\
\hline $\mathrm{N}^{-}$ & 713 & $646(90.6)$ & $67(9.4)$ & \\
\hline $\mathrm{N}^{+}$ & 6 & $4(66.7)$ & $2(33.3)$ & \\
\hline \multicolumn{4}{|c|}{ EGD gross type } & 0.061 \\
\hline I, IIa, IIb & 447 & $440(92.2)$ & $37(7.8)$ & \\
\hline IIc, III & 1426 & $1273(89.3)$ & $153(10.7)$ & \\
\hline \multicolumn{4}{|c|}{ Preoperative WHO type } & $<0.001$ \\
\hline WD & 453 & $438(96.7)$ & $15(3.3)$ & \\
\hline MD & 726 & $643(88.6)$ & $83(11.4)$ & \\
\hline PD & 892 & $783(87.8)$ & $109(12.2)$ & \\
\hline \multicolumn{4}{|l|}{ Lauren type } & $<0.001$ \\
\hline Intestinal & 1220 & $1123(92.0)$ & $97(8.0)$ & \\
\hline Diffuse & 662 & $587(88.7)$ & $75(11.3)$ & \\
\hline Mixed & 159 & $125(78.6)$ & $34(21.4)$ & \\
\hline \multicolumn{4}{|l|}{ Tumor size } & $<0.001$ \\
\hline$<2.5 \mathrm{~cm}$ & 1075 & $1015(94.4)$ & $60(5.6)$ & \\
\hline$\geq 2.5 \mathrm{~cm}$ & 995 & $848(85.2)$ & $147(14.8)$ & \\
\hline \multicolumn{4}{|c|}{ Tumor location (longitudinal) } & 0.189 \\
\hline Upper $1 / 3$ & 192 & $166(86.5)$ & $26(13.5)$ & \\
\hline Middle 1/3 & 581 & $526(90.5)$ & $55(9.5)$ & \\
\hline Lower $1 / 3$ & 1224 & $1109(90.6)$ & $115(9.4)$ & \\
\hline \multicolumn{4}{|c|}{ Tumor location (circular) } & 0.015 \\
\hline $\mathrm{LC}$ & 822 & $753(91.6)$ & $69(8.4)$ & \\
\hline GC & 255 & $216(84.7)$ & $39(15.3)$ & \\
\hline AW & 454 & $410(90.3)$ & $44(9.7)$ & \\
\hline PW & 511 & $462(90.4)$ & 49 (9.6) & \\
\hline
\end{tabular}

$M D C T$ multidetector-row computed tomography, EUS endoscopic ultrasonography, $E G D$ esophagogastroduodenoscopy, $W D$ well-differentiated, $M D$ moderately differentiated, $P D$ poorly differentiated, $L C$ lesser curvature, $G C$ greater curvature, $A W$ anterior wall, $P W$ posterior wall 
Table 4 Multivariable analysis to evaluate potential predictive factors for lymph node metastases (LNM) in clinically early gastric cancer (cEGC) patients

\begin{tabular}{|c|c|c|c|c|c|c|}
\hline \multirow[t]{2}{*}{ Variables } & \multirow[t]{2}{*}{ Prognostic score point } & \multirow[t]{2}{*}{ B } & \multirow[t]{2}{*}{ Significance } & \multirow[t]{2}{*}{$\operatorname{Exp}(B)$} & \multicolumn{2}{|c|}{$95 \%$ CI for $\operatorname{Exp}(B)$} \\
\hline & & & & & Lower & Upper \\
\hline \multicolumn{2}{|c|}{$\mathrm{N}$ stage by MDCT } & 1.389 & $<0.001$ & 4.010 & 2.652 & 6.062 \\
\hline $\mathrm{N}^{-}$ & 0 & & & & & \\
\hline $\mathrm{N}^{+}$ & 4 & & & & & \\
\hline \multicolumn{2}{|c|}{ Preoperative WHO type } & 0.632 & $<0.001$ & 1.881 & 1.433 & 2.469 \\
\hline WD & 0 & & & & & \\
\hline $\mathrm{MD}+\mathrm{PD}$ & 2 & & & & & \\
\hline \multicolumn{2}{|l|}{ Tumor size } & 0.981 & $<0.001$ & 2.666 & 1.937 & 3.669 \\
\hline$<2.5 \mathrm{~cm}$ & 0 & & & & & \\
\hline$\geq 2.5 \mathrm{~cm}$ & 3 & & & & & \\
\hline
\end{tabular}

$M D C T$ multidetector row computed tomography, $W D$ well-differentiated, $M D$ moderately differentiated, $P D$ poorly differentiated

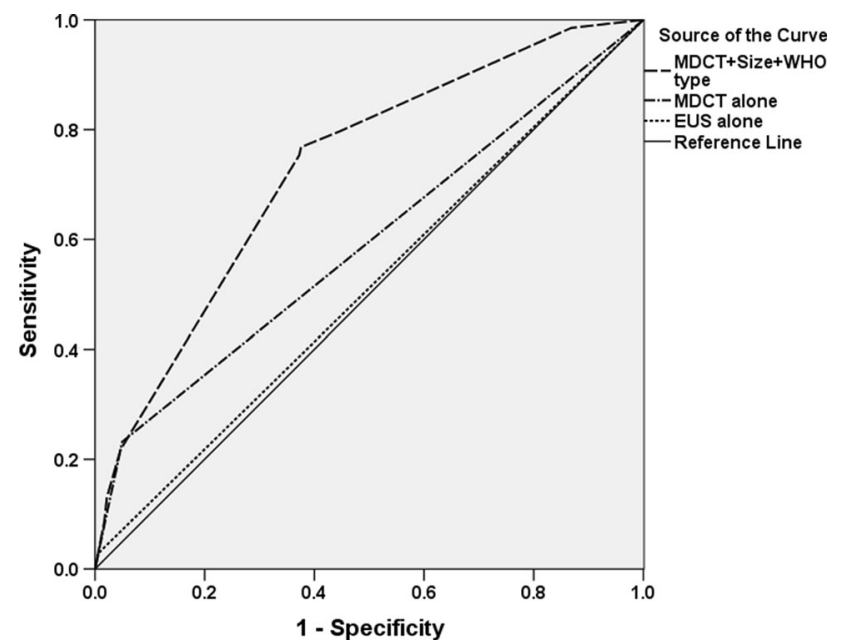

Fig. 2 Comparison of the ROC curve in assessing lymph node metastases by the composite score with multidetector-row computed tomography (MDCT), histological type, and tumor size and diagnosis by MDCT or endoscopic ultrasound (EUS) alone

diagnostic sensitivity of LNM in cEGC, which is significantly better than MDCT or EUS alone.

\section{Relationship between the preoperative composite LNM score system and postoperative pathologically verified $\mathrm{N}$ staging}

As shown in Fig. 3a, according to the composite score of the three variables of $\mathrm{N}$ staging by $\mathrm{CT}$, preoperative $\mathrm{WHO}$ histological type, and tumor size, all cEGC patients were divided into the low-risk group $(<4$ points) and the highrisk group ( $\geq 4$ points) for LNM. Because the current preoperative assessment of LNM relies on conventional diagnostic imaging, the high-risk group was subdivided into two subgroups: the high-risk subgroup A for LNM diagnosed by tumor size and differentiation assessment without diagnosis by MDCT and the high-risk subgroup B for LNM determined by conventional MDCT diagnosis. Using the two staging methods, including the number of positive lymph nodes and the site of LNM, the proportions of each risk group according to the composite score in the $\mathrm{N}$ staging were compared. As shown in Fig. 3b, according to the $\mathrm{N}$ staging criteria of AJCC cancer staging handbook, 7 th edition [8], in the 207 cases of cEGC patients with LNM, the numbers of patients in the low-risk group in stages $\mathrm{N} 1 / \mathrm{N} 2 / \mathrm{N} 3$ were $41 / 9 / 2$, accounting for $32.0 \%$, $15.3 \%$, and $10.0 \%$ of the patients in each stage of LNM, respectively. Comparison of the patients with LNM in the low-risk group and the high-risk group showed that the proportion of $>\mathrm{N} 1$ metastases in the low-risk group $(21.2 \%)$ was significantly lower than in the high-risk group $(43.9 \%)$, and the difference was statistically significant $(P=0.004)$. Among the 207 cases of patients with LNM, the postoperative pathological LNM sites were identified in 143 continuous cases from 2006, when the lymph nodes were examined according to the exact lymph node stations. According to the risk and the LNM sites of the foregoing patients (Fig. 3c), the proportions of the cases in the low-risk group with first-tier and second-tier LNM were $20.2 \%$ and $10.3 \%$, respectively. The proportion of patients with second-tier LNM in the low-risk group $(19.4 \%)$ was significantly lower than in the high-risk group $(43.9 \%)$, and the difference was statistically significant $(P=0.009)$. For the 7 patients with second-tier LNM in the low-risk group, the metastatic sites were located in Station 7 (5 cases), Station 8 ( 1 case), and Station $11 \mathrm{p}$ ( 1 case). In addition, Fig. 3 shows that the proportions of $>\mathrm{N} 1 \mathrm{LNM}$ and second-tier LNM in the patients in the high-risk group A were $40.0 \%$ and $38.8 \%$, respectively, which were significantly higher than the values of $21.2 \%$ and $19.4 \%$ in the low-risk group ( $P=0.017$ and 0.040 , respectively), with no significant difference compared to the metastatic proportions of 
a

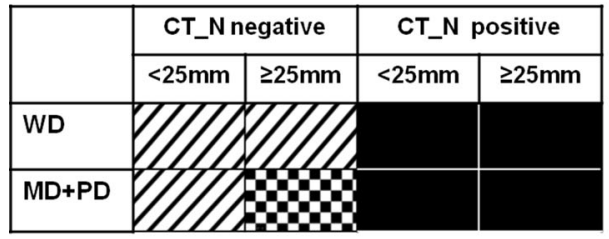

ZLNM low risk $\mathbf{Q}$ LNM high risk A $\square_{\text {LNM high risk B }}$

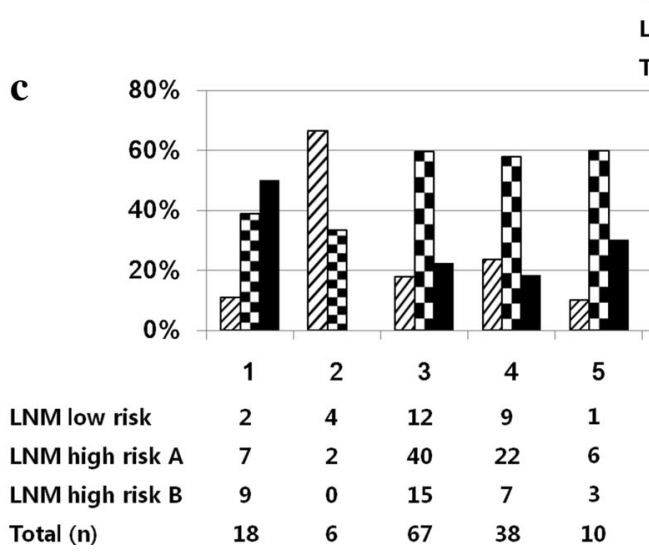

b

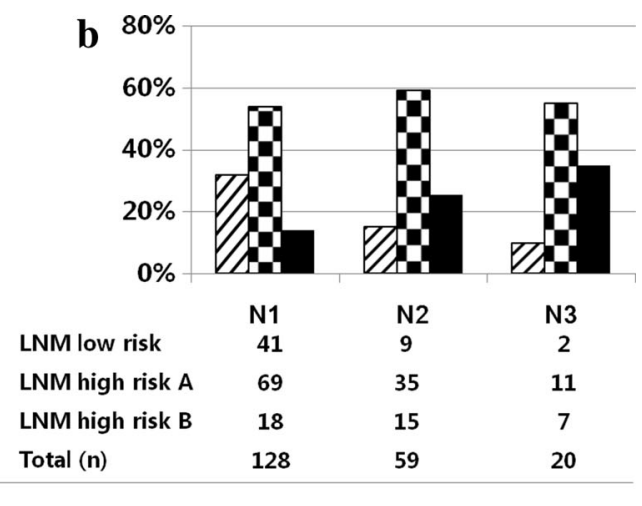

Total (n) 128

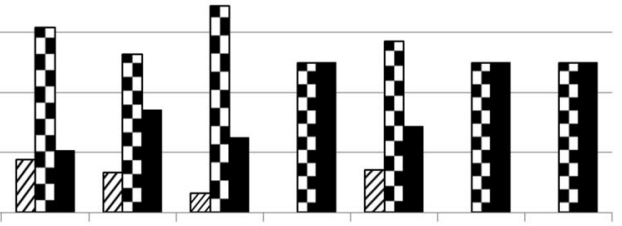

$\begin{array}{ccccccc}6 & 7 & 8 & 9 & 11 p & 11 d & 12 \\ 6 & 5 & 1 & 0 & 1 & 0 & 0 \\ 21 & 20 & 11 & 1 & 4 & 1 & 2 \\ 7 & 13 & 4 & 1 & 2 & 1 & 2 \\ 34 & 38 & 16 & 2 & 7 & 2 & 4\end{array}$

Fig. 3 Relationship of preoperative risk score of lymph node metastases and postoperative N staging. a Grouping based on preoperative assessment of the risk of lymph node metastases. b Distribution of the patients in the high-risk group and the low-risk group according to the $\mathrm{N}$ staging of the AJCC Cancer Staging Handbook, 7th edition. c Distribution of different risk subgroups in each site of lymph node metastases

$55.0 \%$ and $59.3 \%$ in the high-risk group B $(P=0.10$ and 0.063 , respectively).

\section{Comparison of the preoperative WD and MD groups}

Among the selected cEGC patients, 453 and 726 were preoperatively diagnosed WD and MD, respectively, by histopathological examination. Comparison of the preoperative WD and MD groups revealed that the proportions of the patients in the WD group with N1, N2, and N3 were $2.4 \%$ (11/453), $0.9 \%$ (4/453), and $0 \%$ (0/453), respectively, which were significantly lower than the corresponding values of $8.3 \%$ (60/726), $2.5 \%$ (18/726), and $0.7 \%(5 / 726)$ in the MD group $(P<0.001)$ (Supplementary Table 1). In addition, comparison of the preoperative and postoperative histological classification revealed that in patients with the preoperative diagnosis of MD, $12.7 \%$ (92/726) had a postoperative pathological diagnosis of undifferentiated type, which was significantly higher than $0.9 \%(4 / 453)$ of the WD patients $(P<0.001)$ (Supplementary Table 1). In the group of tumors with diameter $\geq 2.5 \mathrm{~cm}$, the proportion of cases with preoperative biopsy of differentiated type and postoperative pathological diagnosis of undifferentiated type $(11.1 \%, 58 / 524)$ was significantly higher than in the group of tumors with diameter $<2.5 \mathrm{~cm}(5.8 \%, 38 / 655)(P<0.001)$ (Supplementary Table 2).

\section{Discussion}

With the development of minimally invasive surgical techniques, the accuracy and safety of gastric surgery have received more and more attention. Although D2 lymph node dissection is still the mainstream surgical treatment of gastric cancer, for EGC patients without LNM or with confined LNM, an excessive dissection of the lymph node will not only fail to improve the prognosis of patients but also will increase the possibility of postoperative complications. Therefore, Japanese scholars proposed that patients with a preoperative diagnosis of $\mathrm{cNO}$ early gastric cancer should be able to choose $\mathrm{D} 1^{+}$lymph node dissection, that is, the modified D2 dissection [12], which sets a strong requirement for precise preoperative $\mathrm{T}$ staging and the assessment of LNM in gastric cancer. To date, with the combination of endoscopy, MDCT, and EUS examinations, the accuracy of the preoperative diagnosis of EGC has reached $76.2 \%$ to $96.8 \%$ [13-15]. However, some cases of advanced gastric cancer (AGC) were still preoperatively diagnosed as EGC. For these AGC patients with underestimation in preoperative diagnosis, in certain cases 
including pT3, pT4, and some pT2 patients, the actual situation could be revealed by inspecting the resected specimen during the surgery, and the original surgical treatment method could be changed. Other cases, however, especially for pT2 patients whose tumor is infiltrating the muscular propria, some patients were often treated as EGC because of the difficulty in distinguishing the tumor lesions and the pT1 gastric carcinoma by the naked eye. This situation is more likely to occur in laparoscopic surgery for gastric cancer, in which the manipulation of the tumor is limited [16]. Whether underestimation of preoperative $\mathrm{T}$ staging will affect the long-term prognosis in patients with cT1pT2 gastric cancer remains unclear. Tokunaga et al. [17] reported that the postoperative 5-year survival rate in patients with cT1pT2 gastric cancer was significantly better than in patients with cT2-4pT2 gastric cancer. However, the $\mathrm{T}$ stage of that study was based on the staging criteria of the AJCC cancer staging handbook, 6th edition, in which the T2 stage included cases with invasion in the muscularis propria (T2a) and submucosa (T2b). The proportion of T2b patients in the cT2-4pT2 group was significantly higher than in the cT1pT2 group $(58.1 \%$ vs. $28.6 \%$ ), and the difference in the long-term survival between the cT1pT2 group and the pathological EGC group was not compared in that study. Therefore, the present research took the initiative by comparing the pT2 (cT1pT2) patients with other two groups (cT1pT1 and cT2$4 \mathrm{pT} 2$ ). By comparing the survival curves, it was found that the 5-year survival rates of the patients in the cT1pT2 group and the cT1pT1 group were not significantly different $(P=0.059)$ but were significantly higher than the survival rate of the patients in the cT2-4pT2 group (92.5\% vs. $78.0 \%, P<0.001)$. Although the $P$ value between the cT1pT2 group and the cT1pT1 group was close to 0.05 , it should be considered not significant because of the large sample size of the present series. In the meantime, the survival curve between the cT1pT2 group and the cT24 pT2 was significantly different $(P<0.001)$. The foregoing results were supported by an associated study [17]. It is reasonable to believe cT1pT2 patients have similar longterm prognosis with ECG but not AGC. Patients with cT1pT2 can be treated the same as EGC patients receiving selective localized lymph node dissection. Further multivariate analysis showed that LNM was the independent risk factor with an impact on the long-term prognosis for patients in cEGC. These results suggest that for cEGC patients in stages pT1 and pT2, the difference in T staging before and after surgery does not affect the long-term prognosis of surgical treatment, and accurate preoperative assessment of LNM is the key to the long-term efficacy of surgical treatment.

The current preoperative assessment of LNM primarily relies on MDCT and other imaging studies. However, compared to $\mathrm{T}$ staging, the assessment result of preoperative $\mathrm{N}$ staging is unsatisfactory. A recent meta-analysis study on the preoperative assessment of LNM of gastric cancer [18] showed that the existing diagnostic methods, including abdominal ultrasonography (AUS), EUS, MDCT, conventional magnetic resonance imaging (MRI), and ${ }^{18} \mathrm{~F}$-fluoro-2-deoxyglucose positron emission tomography (FDG-PET), cannot be reliably used to confirm or exclude the presence of LNM, the main reason being that the current radiologic diagnosis of LNM is primarily based on changes in the size of lymph nodes. However, a survey on the relationship between the size of lymph node and metastatic infiltration showed that, even though the diameter of $80 \%$ of lymph nodes with no tumor invasion was less than or equal to $5 \mathrm{~mm}$, the diameter of only $45 \%$ of lymph nodes with metastatic invasion was larger than $5 \mathrm{~mm}$ [19]. Especially for EGC, the chance of enlarged lymph nodes from tumor metastatic invasion is low, which greatly affects the sensitivity of the preoperative diagnosis of LNM. In this study, the sensitivity of preoperative diagnosis of LNM in cEGC by MDCT was only $19.3 \%$. Compared with MDCT, the sensitivity of EUS is even lower, only $2.9 \%$. This low sensitivity might also be related to the fact that EUS has a limited depth of penetration, so that the visualization of distant lymph nodes is difficult. According to the previous studies, many tumor characteristics are closely associated with the metastases of lymph nodes in EGC [6], and these tumor characteristics can be accurately revealed before the surgery. Currently, these tumor characteristics have become the main relevant indicators in identifying gastric cancer patients who are suitable for receiving ESD treatment [20]. Whether these tumor characteristics can also serve as a diagnostic indicator for preoperatively assessing the extent of lymph node dissection in gastric cancer radical surgery remain unclear. To answer this question, the relevant preoperative indicators were selected in this study for univariate and multivariate analyses to discover the tumor characteristics related to LNM, thereby enhancing the sensitivity of the preoperative diagnosis of LNM. The results indicate that the three variables in $\mathrm{N}$ stage by $\mathrm{CT}$, preoperative $\mathrm{WHO}$ histological type, and tumor size are the independent risk factors of LNM in cEGC. A composite scoring criterion established with these three variables showed $74.7 \%$ sensitivity in predicting the occurrence of LNM, which was significantly higher than the sensitivity when relying solely on imaging.

The current study excluded the preoperative diagnosed cT1a and cT1b from the influencing factors in predicting the LNM risk. As the factors used to predict LNM in the current research all come from preoperative examinations, routine preoperative MDCT and endoscopic examinations currently cannot accurately distinguish between T1a and 
T1b. Even by endoscopic ultrasonography, the accuracy is only $67.4 \%(64.4-70.4 \%)$ [21] for T staging of EGC. Therefore, the subgroups of cEGC (cT1a and cT1b) were not included in the analysis of predicting LNM.

In contrast to the previous related studies that adopted a postoperative histological classification with differentiated and undifferentiated types $[22,23]$, this study divided the preoperative histological classification into WD and $\mathrm{MD}+\mathrm{PD}$ groups in the multivariate analysis because of the similar incidence of LNM between preoperative MD and PD groups. According to the results from comparing the difference between preoperative $\mathrm{WD}$ and $\mathrm{MD}$, the probability of LNM in the preoperative MD group was significantly higher than in the WD group, and in addition, a considerable number of patients with undifferentiated type might be preoperatively underestimated as MD. Additionally, with increasing tumor diameter, this diagnostic error gradually increases. Based on these concerns, this study combined both preoperatively $\mathrm{MD}$ and $\mathrm{PD}$ patients into one group to predict the risk of LNM.

Based on the comprehensive assessment criteria with CT diagnosis of tumor characteristics, the sensitivity of the preoperative prediction of LNM was greatly increased, but LNM still occurred in a considerable number of cEGC patients in the low-risk group of LNM (52/1138). For these patients, whether limited lymph node dissection can achieve a radical treatment result remains unclear. Further detailed analysis of the total number and positions of lymph node metastases in the patients with LNM showed that, in the low-risk group, 1 or 2 metastatic lymph nodes in the N1 stage (41/52) commonly occurred, which were mostly confined to the first-tier lymph nodes of the gastric periphery. For patients with second-tier LNM in the lowrisk group, the sites of metastases also mainly occurred in the Station 7 lymph nodes; therefore, $\mathrm{D}^{+}{ }^{+}$lymph node dissection can achieve the radical treatment result. Based on these results, we believe that for the patients with a composite score of the risk of LNM $<4$ in the LNM lowrisk group, $\mathrm{D}^{+}{ }^{+}$lymph node dissection is the preferred option; for the patients with a score $\geq 4$ in the high-risk group, standard D2 lymph node dissection is recommended. The foregoing criteria are slightly different from the standard gastric treatment guidelines by the Japanese Gastric Cancer Association (JGCA). In the JGCA guidelines, the preoperative $\mathrm{cN}$ radiographic staging serves as the sole criterion for $\mathrm{D}^{+}$or $\mathrm{D} 2$ lymph node dissection in cEGC: the patients in the $\mathrm{cN}^{-}$stage undergo $\mathrm{D} 1$ or $\mathrm{D} 1^{+}$ dissection, whereas the patients in the $\mathrm{cN}^{+}$stage undergo D2 dissection [12]. However, our study found that among the cEGC patients who showed no LNM by preoperative $\mathrm{CT}$, but had a preoperative diagnosis of MD or PD, and had a tumor diameter greater than $2.5 \mathrm{~cm}$, there was a high incidence of LNM. The proportion of distant LNM was comparable to the proportion among the patients who showed LNM by CT. The proportion of LNM in $>\mathrm{N} 1$ stage was up to $40.0 \%$, of which $38.8 \%$ had the LNM in the second tier, and many of the LNM sites were located in Station 11d and Station 12 under the coverage of D2 dissection. Therefore, in addition to the preoperative diagnosis of LNM by MDCT, the tumor size and its histological type should also be used as references to decide the range of the lymph node dissection.

In summary, for cEGC patients with the postoperative diagnosis of pT1 and pT2 stage, LNM is the most important prognostic factor. A preoperative comprehensive assessment of the risk of LNM according the tumor size, preoperative differentiation type, and $\mathrm{N}$ staging by $\mathrm{CT}$ is a simple and effective method to decide the reasonable range of lymph node dissection in clinical practice. However, this study is a retrospective study, and the tumor size data used in this study were the maximum diameters of the tumors in the postoperative pathological measurement. In practice, it is difficult to accurately measure the maximum diameter of the tumor by preoperative endoscopy or CT. Therefore, the feasibility of these assessment criteria requires further validation in future prospective studies. Some observational studies from high-volume centers using prospectively collected data could be useful to validate the present scoring system predicting nodal metastases in cEGC.

\section{Compliance with ethical standards}

Conflict of interest No conflict of interest exists.

\section{References}

1. Shen L, Shan YS, Hu HM, Price TJ, Sirohi B, Yeh KH, et al. Management of gastric cancer in Asia: resource-stratified guidelines. Lancet Oncol. 2013;14:e535-47.

2. Koeda K, Nishizuka S, Wakabayashi G. Minimally invasive surgery for gastric cancer: the future standard of care. World J Surg. 2011;35:1469-77.

3. Allum WH. Optimal surgery for gastric cancer: is more always better? Recent Result Cancer Res. 2012;196:215-27.

4. Kwee RM, Kwee TC. Imaging in local staging of gastric cancer: a systematic review. J Clin Oncol. 2007;25:2107-16.

5. Yoshikawa T, Ishiwa N, Morinaga S, Noguchi Y, Yamamoto Y. Can surgical diagnosis of "early" gastric cancer and lymph node metastasis be accurate? Gastric Cancer. 2004;7:36-40.

6. Kwee RM, Kwee TC. Predicting lymph node status in early gastric cancer. Gastric Cancer. 2008;11:134-48.

7. Ahn HS, Lee HJ, Yoo MW, Kim SG, Im JP, Kim SH, et al. Diagnostic accuracy of $\mathrm{T}$ and $\mathrm{N}$ stages with endoscopy, stomach protocol CT, and endoscopic ultrasonography in early gastric cancer. J Surg Oncol. 2009;99:20-7.

8. Edge SB, Byrd DR, Compton CC, Fritz AG, Greene FL, Trotti A. AJCC cancer staging handbook. 7th ed. Chicago: American Joint Committee on Cancer; 2010. 
9. Association Japanese Gastric Cancer. Japanese classification of gastric carcinoma: 2nd English edition. Gastric Cancer. 1998;1:10-24.

10. Rassi A Jr, Rassi A, Little WC, Xavier SS, Rassi SG, Rassi AG, et al. Development and validation of a risk score for predicting death in Chagas' heart disease. N Engl J Med. 2006;355:799-808.

11. Du C, Zhou Y, Huang K, Zhao G, Fu H, Shi Y. Defining a high-risk subgroup of pathological T2N0 gastric cancer by prognostic risk stratification for adjuvant therapy. J Gastrointest Surg. 2011;15:2153-8.

12. Association Japanese Gastric Cancer. Japanese gastric cancer treatment guidelines 2010 (ver. 3). Gastric Cancer. 2011;14:113-23.

13. Choi J, Kim SG, Im JP, Kim JS, Jung HC, Song IS. Comparison of endoscopic ultrasonography and conventional endoscopy for prediction of depth of tumor invasion in early gastric cancer. Endoscopy. 2010;42:705-13.

14. Hwang SW, Lee DH, Lee SH, Park YS, Hwang JH, Kim JW, et al. Preoperative staging of gastric cancer by endoscopic ultrasonography and multidetector-row computed tomography. J Gastroenterol Hepatol. 2010;25:512-8.

15. Feng XY, Wang W, Luo GY, Wu J, Zhou ZW, Li W, et al. Comparison of endoscopic ultrasonography and multislice spiral computed tomography for the preoperative staging of gastric cancer-results of a single institution study of 610 Chinese patients. PLoS One. 2013;8:e78846.

16. Lee HH, Yoo HM, Song KY, Jeon HM, Park CH. Risk of limited lymph node dissection in patients with clinically early gastric cancer: indications of extended lymph node dissection for early gastric cancer. Ann Surg Oncol. 2013;20:3534-40.

17. Tokunaga M, Hiki N, Fukunaga $T$, Ohyama S, Yamada K, Yamaguchi T. Better prognosis of $\mathrm{T} 2$ gastric cancer with preoperative diagnosis of early gastric cancer. Ann Surg Oncol. 2009;16:1514-9.

18. Kwee RM, Kwee TC. Imaging in assessing lymph node status in gastric cancer. Gastric Cancer. 2009;12:6-22.

19. Monig SP, Zirbes TK, Schroder W, Baldus SE, Lindemann DG, Dienes HP, et al. Staging of gastric cancer: correlation of lymph node size and metastatic infiltration. AJR Am J Roentgenol. 1999;173:365-7.

20. Shim CN, Lee SK. Endoscopic submucosal dissection for undifferentiated-type early gastric cancer: do we have enough data to support this? World J Gastroenterol. 2014;20:3938-49.

21. Choi J, Kim SG, Im JP, Kim JS, Jung HC, Song IS. Comparison of endoscopic ultrasonography and conventional endoscopy for prediction of depth of tumor invasion in early gastric cancer. Endoscopy. 2010;42:705-13.

22. Min BH, Kim KM, Park CK, Lee JH, Rhee PL, Rhee JC, et al. Outcomes of endoscopic submucosal dissection for differentiated-type early gastric cancer with histological heterogeneity. Gastric Cancer. 2014; doi:10.1007/s10120-014-0378-7.

23. Kim JH, Kim YH, da Jung H, Jeon HH, Lee YC, Lee H, et al. Follow-up outcomes of endoscopic resection for early gastric cancer with undifferentiated histology. Surg Endosc. 2014;28:2627-33. 